\title{
Modelling of Light Trapping in Acidic-Textured Multicrystalline Silicon Wafers
}

\author{
Yang Li, Zhongtian Li, Yuebin Zhao, and Alison Lennon \\ School of Photovoltaic and Renewable Energy Engineering, The University of New South Wales, Sydney, NSW 2052, Australia \\ Correspondence should be addressed to Alison Lennon, a.lennon@unsw.edu.au
}

Received 31 October 2011; Accepted 6 November 2011

Academic Editor: Donghwan Kim

Copyright () 2012 Yang Li et al. This is an open access article distributed under the Creative Commons Attribution License, which permits unrestricted use, distribution, and reproduction in any medium, provided the original work is properly cited.

Acidic texturing has been widely used to reduce the reflection losses for silicon solar cells fabricated on multicrystalline wafers, however, there are few available models which attempt to predict the reduced reflection after texturing based on the morphology of the textured surfaces. An optical model which simulates the light trapping and scattering effects of acidic-textured surfaces based on the surface morphology is presented. The developed model was experimentally verified by reflection measurements from multicrystalline silicon wafers textured using different etching conditions. The relationship between weighted average reflection and surface morphology is demonstrated with some of the trends being explained by simulating reflection in different wavelength regions. The developed model could be embedded into solar cell simulation tools or adapted to predict optical properties of diverse surface morphologies.

\section{Introduction}

Surface texturing is a key process in the fabrication of silicon solar cells both in the laboratory and in commercial production. It provides enhanced light absorption with less front surface reflection, more internal reflection, and increased light path lengths inside the cell $[1,2]$. A wide range of physical and chemical methods have been employed for texturing of silicon wafer-based and thin-film solar cells, including lithography $[3,4]$, mechanical grooving $[5,6]$, reactive ion texturing [7-9], laser texturing [10-12], alkaline [13-15], and acidic texturing [16-20].

Of these texturing methods, alkaline and acidic texturing have been widely adopted in the commercial production of silicon wafer-based solar cells due to their lower cost, amenability to high-throughput processing, and superior antireflection properties. Alkaline etchants etch the $\{100\}$ planes of silicon much faster than the $\{111\}$ planes. This anisotropic etching, when performed at low sodium or potassium hydroxide concentrations and temperatures of $80-90^{\circ} \mathrm{C}$, results in the formation of pyramidal structures on $\{100\}$ orientated monocrystalline silicon. These pyramidal structures trap the incoming light and reduce reflection effectively [2]. However, alkaline texturing performed on multicrystalline silicon results in largely untextured regions (where $\{111\}$ crystal planes are exposed at the surface) and steps between grains due to the different grain orientations of the multicrystalline silicon $[15,21,22]$. Although acidic texturing of multicrystalline wafers results in higher average reflectance values than typically observed for alkaline texturing of monocrystalline wafers, the acidic texturing process is relatively low-cost and can be effectively performed as an inline process in a manufacturing line. In fact, because of the ease of implementing acidic texturing compared to alkaline texturing, some silicon solar cell manufacturers select to also texture monocrystalline wafers using acidic texturing, because the difference in surface reflection between alkalineand acidic-textured surfaces is reduced significantly after application of a silicon nitride antireflection coating and encapsulation.

Acidic texturing has also been employed to texture $\mathrm{ZnO}$ : Al transparent conducting oxide (TCO) layers, which serve as window layers in silicon-based thin-film solar cells [23]. It may also find application in the texturing of wafers produced using seeded casting processes [24]. The wafers that result from these new casting processes still contain 
grains but have large regions of monocrystalline material. A number of different methods are being developed for largescale manufacture of this new silicon material [24-26], and because it is likely that grain orientation cannot always be carefully controlled, acidic texturing may also find a role in texturing these new wafer surfaces.

Simulation tools, such as PC1D [27], contain models that simulate the reflection and light trapping for alkalinetextured solar cells and predict the electrical performance of textured solar cell devices. These tools enable higher level solar cell production line simulators, such as the Virtual Production Line (VPL), which has been developed at the University of New South Wales (UNSW) [28, 29], to relate the effects of different alkaline texturing conditions (e.g., chemical composition, time, and temperature) on final device electrical performance. The VPL simulation software is extensively used in the teaching of photovoltaic engineering at UNSW. However, the current version of this software can only simulate the reflectance of alkalinetextured surfaces, which limits its application to current manufacturing processes which involve the use of multicrystalline silicon wafers.

Although the chemical process for acidic texturing can be simulated [29], a model is required to predict the reflectance as a function of wavelength and hence the electrical performance of the textured solar cell device. The light-trapping properties of the acidic-textured surface morphology have not been extensively studied. Earlier studies have considered only the front-surface reflection properties of these surfaces [21, 22]. However, this paper describes a more comprehensive model which considers the reflection from the entire solar power spectrum, bulk absorption, and the contribution of the rear surface to light trapping. These enhancements to the model become critical as thinner wafers are increasingly used in the manufacturing of cells. Acidic texturing experiments were performed and the measured reflectance results were compared to those predicted by the model. The results suggest that the model can predict the reflection for a wide range of wavelengths. Further work is required to integrate this reflection model in solar cell simulators such as PC1D or higher level simulators such as VPL.

\section{Model of Acidic-Textured Surfaces}

Multicrystalline silicon surfaces textured using the UKN recipe for acidic texturing [18-20] are typified by an irregular array of concavities, or etching pits, as shown in Figure 1. These pits in the textured surface are generated by the etching of surface defects in the crystal structure caused by sawing damage. Unlike in alkaline texturing where an initial saw-damage etching process must be performed before the texturing, in acidic texturing the saw damage is removed during the texturing thus simplifying the manufacturing process. However, the weighted average reflectance (WAR) values achieved by alkaline texturing are significantly lower than those achieved with acidic texturing. Furthermore, if acidic texturing times are used that are longer than optimal,

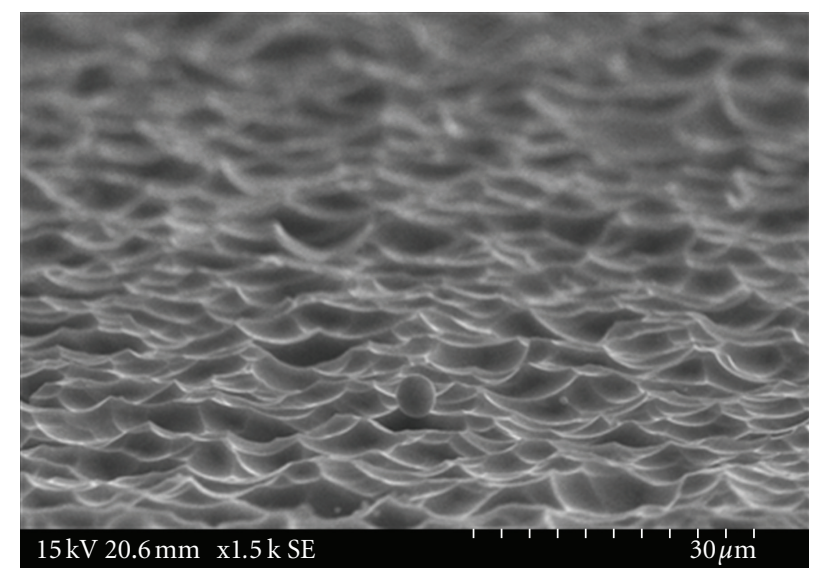

Figure 1: A scanning electron microscope (SEM) image of an acidic-textured multicrystalline silicon wafer surface showing the formation of randomly arranged etching pits on the surface. The surface was textured for 2 mins at $10^{\circ} \mathrm{C}$ in a solution comprising an $\mathrm{HF}: \mathrm{HNO}_{3}: \mathrm{H}_{2} \mathrm{O}$ ratio of $15: 50: 35$.

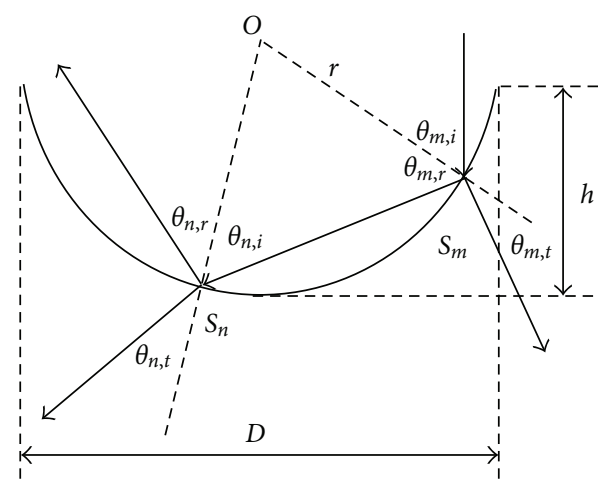

FIgURE 2: Schematic depicting a cross-section of a spherical cap, and the associated variables, used to model the etch pits of an acidictextured surface.

then the textured concavities become larger and flatter and the resulting surface is significantly more reflective [30].

To simplify the simulation of the reflection based on surface morphology described in this paper, a spherical cap surface was used to model the etching pit structure of the acidic-textured surface. The front-surface reflection, bulk absorption, rear surface reflection, and further internal reflections of light reaching each part of the etching pit were estimated, with the reflection of the wafer being estimated as the weighted average of all components.

2.1. Front Surface Scattering. The spherical model is shown schematically in Figure 2. The variables $O$ and $r$ denote the centre and radius of the sphere cap, and $D$ and $h$ donate the diameter and height of the sphere cap, respectively. The sphere cap is divided into small annular surface elements, $S_{1}, S_{2}, \ldots, S_{m}, \ldots, S_{N}$, each element having a subtended angle $d \theta$ to $O$. The $k$ th-order reflection at the $m$ th annular surface element is denoted using $R_{m, k}$. 
It is assumed that incident light is directed perpendicularly to the silicon surface, and hence also perpendicular to the base of the sphere cap. Considering the incident light reaching annular surface element $S_{m}$ with an incident angle of $\theta_{m, i}$ and a corresponding refracted angle of $\theta_{m, t}$, the primary reflection at the $m$ th annular surface element $S_{m}$ is given by

$$
R_{m, 1}=\frac{R_{\mathrm{TE} m, 1}+R_{\mathrm{TM} m, 1}}{2},
$$

where $R_{\mathrm{TE}}$ and $R_{\mathrm{TM}}$ represent the reflection of the transverseelectric and transverse-magnetic waves of the incident light, respectively. Light rays having a large incident angle can be reflected more than once as shown in Figure 2. The reflected ray intersects the sphere again at annular surface element $S_{n}$ with the incident angle, $\theta_{n, i}$ and refracted angle $\theta_{n, t}$, which are equal to $\theta_{m, i}$ and $\theta_{m, t}$, respectively, before being reflected away from the surface. Thus, the front surface reflection $R_{F m}$ for the $m$ th annular surface element is given by

$$
\begin{aligned}
R_{F m} & =\prod_{k=1}^{k_{m}} R_{F m, k} \\
& =\left\{\frac{1}{2}\left[\frac{\sin ^{2}\left(\theta_{m, t}-\theta_{m, i}\right)}{\sin ^{2}\left(\theta_{m, t}+\theta_{m, i}\right)}+\frac{\tan ^{2}\left(\theta_{m, t}-\theta_{m, i}\right)}{\tan ^{2}\left(\theta_{m, t}+\theta_{m, i}\right)}\right]\right\}^{k_{m}},
\end{aligned}
$$

where $k_{m}$ donates the highest reflection order and is given by

$$
k_{m}=\left\lfloor\frac{\pi}{\pi-2 \theta_{m, i}}\right\rfloor .
$$

The expression in (3) is derived from internal angle sum of the polygon, which consists of the intersection points and $O$ as its vertices.

2.2. Bulk Absorption. Considering the same incident light ray that reached $S_{m}$ discussed above, the attenuation factor $A_{m}$ after being absorbed inside the wafer is given by

$$
A_{m}=e^{-\alpha x}=e^{-\alpha t / \cos \theta_{m, n}},
$$

where $x$ denotes the actual light path length, $t$ is the thickness of the wafer, $\alpha$ is the absorption coefficient, and $\theta_{m, n}$ is the refracted angle of light reaching annular surface element $S_{m}$ referring to the normal direction to the wafer, as shown in Figure 3 . This expression shows that the light path is increased due to the textured surface and hence the probability of absorption by the silicon wafer is increased for the light's first pass through the wafer.

2.3. Rear Surface Reflection. The rear surface is modelled using an inverted sphere cap as shown in Figure 4. The incident light reaching $S_{m}$ on the front surface is scattered with an angle of $\theta_{m, n}$ and has the possibility of reaching the convex sphere surface at the rear of the textured wafer. To evaluate the angle distribution function (ADF) of the rear incident angles, the convex surface of the rear etch

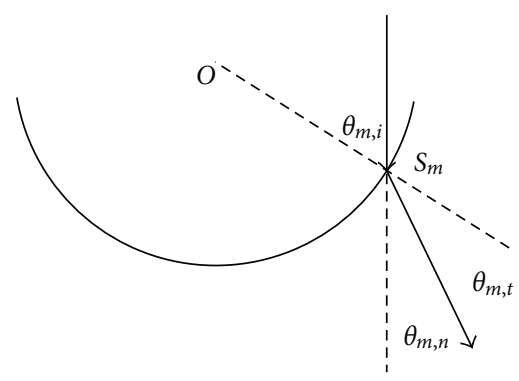

FIGURE 3: Schematic showing the path of a representative transmitted light ray, $\theta_{m, t}$ scattered within the wafer.

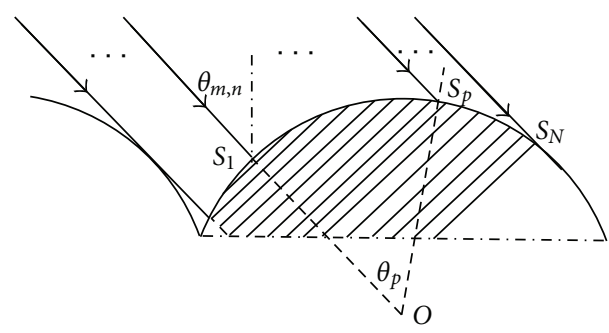

FIGURE 4: Schematic showing parallel light rays incident on the rear acidic-textured surface.

pit is divided into annular surface elements perpendicular to the incident light so, for example, light rays reaching different points on $p$ th annular surface element $S_{p}$ would have the same incident angle of $\theta_{p}$. The ADF is determined by the projection area of the annular surface elements to the incident direction as represented by

$\operatorname{ADF}\left(\theta_{p}\right)=\frac{d S_{p}}{\sum_{p=1}^{N} d S_{p}}=\frac{\left(1-\eta_{p}\right) \pi r_{r}^{2}\left(\sin ^{2} \theta_{p+1}-\sin ^{2} \theta_{p}\right) G_{p}}{\sum_{p=1}^{N} d S_{p}}$,

where $d S_{p}$ is the projected area of unshaded part of $p$ th rear annular surface element, $\eta_{p}$ is the shading percentage of it induced by the adjacent etching pit derived from Figure $4, r_{r}$ is the radius of the sphere cap on the rear surface, and $G_{p}$ is a geometric factor determining the fraction of annular surface element of the sphere cap exposed in the incident direction. The rear surface reflection $R_{R m}$ of the light, which entered via the $m$ th annular front surface element, is the average of the light reflected from the convex surface of the weighted by the $\mathrm{ADF}$ as represented by

$$
R_{R m}=\sum_{p=1}^{N} R\left(\theta_{p}\right) \operatorname{ADF}\left(\theta_{p}\right)
$$

Based on the primary reflection and absorption calculated above and the assumption that these values remain the same during internal reflection, the overall reflection of incident 


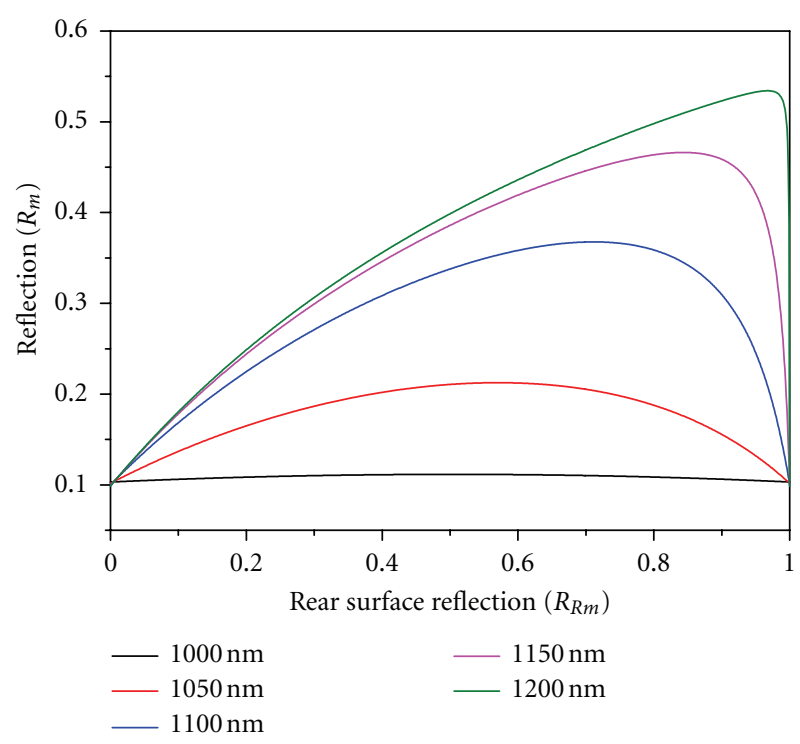

FIgURE 5: Reflection, $R_{m}$, as a function of rear surface reflection, $R_{R m}$, for different wavelengths of incident light. The values of $h / D$ and wafer thickness used in simulation were 0.25 and $200 \mu \mathrm{m}$ respectively.

light reaching $S_{m}$ at the front surface of a free standing acidictextured bare (i.e., uncoated) silicon wafer can be expressed as

$$
\begin{aligned}
R_{m}= & R_{F m}+\left(1-R_{F m}\right) R_{R m}\left(1-R_{R m}\right) A_{m}^{2} \\
& +\left(1-R_{F m}\right) R_{R m}^{3}\left(1-R_{R m}\right) A_{m}^{4}+\cdots \\
= & R_{F m}+\frac{\left(1-R_{F m}\right)\left(1-R_{R m}\right) R_{R m} A_{m}^{2}}{1-R_{R m}^{2} A_{m}^{2}} .
\end{aligned}
$$

Figure 5 depicts the dependence of $R_{m}$ on $R_{R m}$. The value of $R_{m}$ for wavelengths shorter than $1000 \mathrm{~nm}$ does not change with increasing values of $R_{R m}$ due to high absorption coefficient of this light and hence the high probability that this light is absorbed before it reaches the rear surface. However, for longer wavelengths of incident light, $R_{m}$ first increases and then decreases with increasing $R_{R m}$, with a very abrupt decrease being predicted for wavelengths of $\geq 1200 \mathrm{~nm}$. This increase in $R_{m}$ as $R_{R m}$ increases for longer wavelength light is due to the light, which is reflected from the rear surface, ultimately escaping from the wafer before absorption. This reflected loss of initially captured light is more likely for longer wavelength light, which has lower absorption coefficient and is more likely to encounter internal surfaces during its light path through the wafer. It is also enhanced for surfaces which are characterised by lower $h / D$ values where light is coupled less obliquely into the wafer.

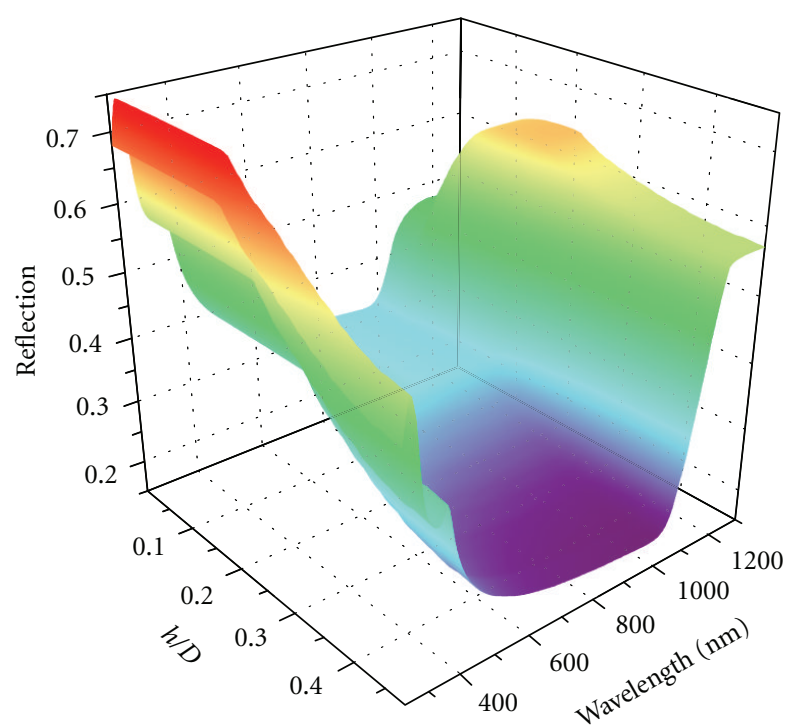

FIGURE 6: Reflection predicted by the model as a function of wavelength and surface morphology indictor $h / D$. The wafer thickness used in the simulations was $200 \mu \mathrm{m}$.

The overall reflection of the etching pit, $R$, is the average reflection of all annular surface elements weighted by their projection area, as given by

$$
\begin{aligned}
R & =\frac{1}{S} \sum_{m=1}^{N} R_{m} d S \\
& =\frac{1}{\pi(D / 2)^{2}} \sum_{m=1}^{N} R_{m} \pi r_{f}^{2}\left(\sin ^{2} \theta_{m+1, i}-\sin ^{2} \theta_{m, i}\right),
\end{aligned}
$$

where $d S$ and $S$ are the projected areas of the small annular surface element and sphere cap, respectively, and $r_{f}$ is the radius of the sphere cap on the front surface, which is not necessarily the same as $r_{r}$ due to processes like rear etching. Figure 6 shows the reflection calculated from (8) as a function of wavelength and $h / D$ ratio. The calculations use light absorption coefficients and refractive indices at $300 \mathrm{~K}$ provided in [31].

\section{Experimental}

Texturing experiments were performed to examine the accuracy of the developed model. In order to show the relationship between reflection and surface morphology, multicrystalline silicon wafers of thickness $200 \mu \mathrm{m}$ were textured in solutions comprising $\mathrm{HF}, \mathrm{HNO}_{3}$, and deionised water to achieve a range of etching pit scales by changing the etching conditions (e.g., chemical composition of the solution and reaction temperature).

The dimensions of the spherical cap (i.e., $h$ and $D$ ) were estimated from SEM cross-sectional images taken from different locations through the wafer. For each wafer surface, values of $h$ and $D$ were estimated as the average of $\sim 20$ measurement points taken from five different SEM crosssectional images. The reflection from the front surface of 


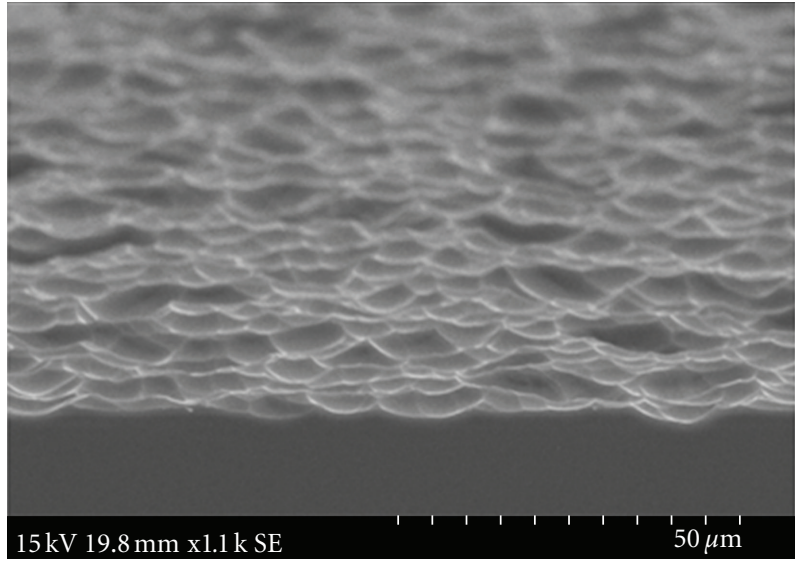

(a)

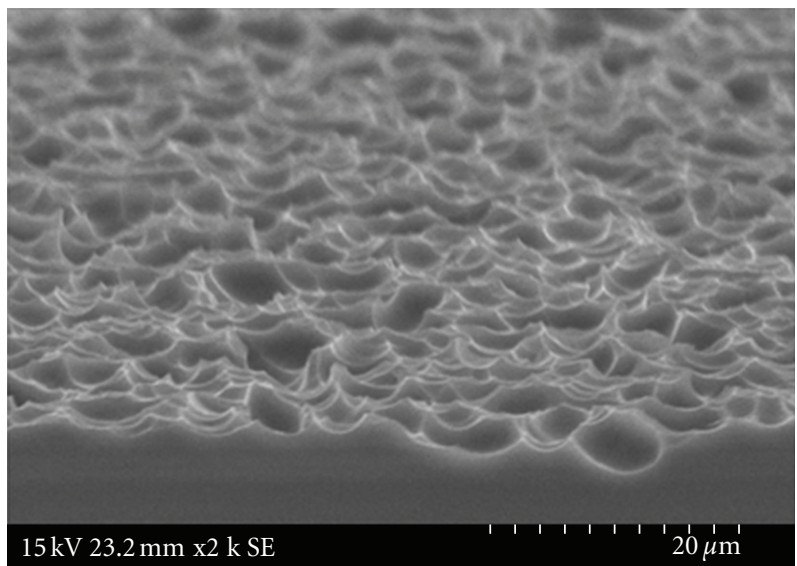

(c)

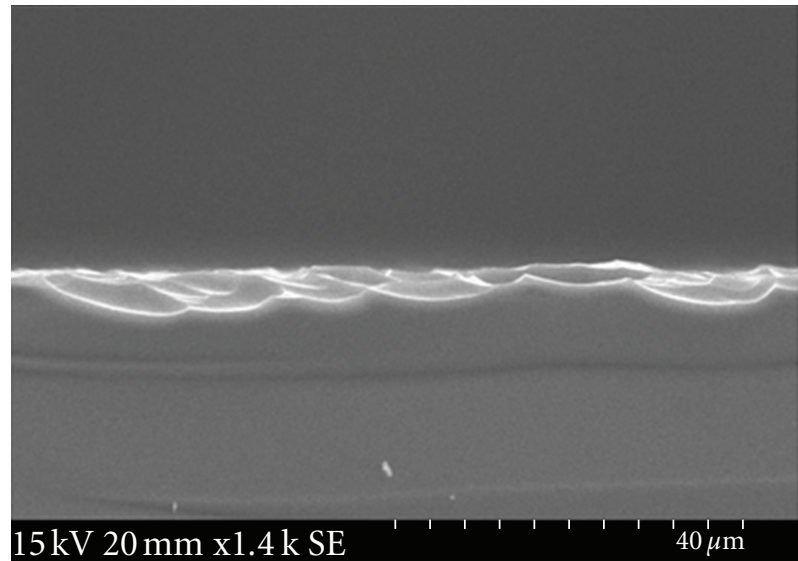

(b)

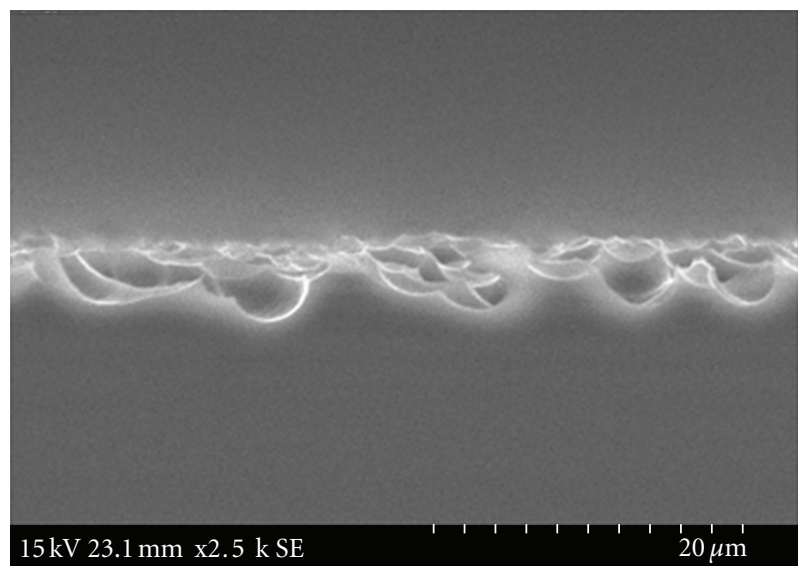

(d)

FIGURE 7: Scanning electron microscope images of the surface and cross sections of multicrystalline silicon wafer surfaces textured under two different conditions: (a,b) $\mathrm{HF}: \mathrm{HNO}_{3}: \mathrm{H}_{2} \mathrm{O}=15: 50: 35,25^{\circ} \mathrm{C}, 2 \mathrm{~min}$; (c,d) $\mathrm{HF}: \mathrm{HNO}_{3}: \mathrm{H}_{2} \mathrm{O}=24: 40: 36,10^{\circ} \mathrm{C}, 2 \mathrm{~min}$.

wafers, which had been textured on both sides, was measured for a wavelength range of $250 \mathrm{~nm}$ to $1300 \mathrm{~nm}$ using a Perkin Elmer spectrophotometer. It was estimated that $<1 \%$ of the light that was transmitted through the wafer was reflected back into the wafer. Weighted average reflection values were calculated by weighting the measured reflection against the intensity of direct radiation in the AM1.5G spectrum.

\section{Results and Discussion}

Figure 7 shows SEM images of some of the different wafer surface morphologies that were observed after acidic texturing. It demonstrates how different etch pit geometries can be achieved using different etching conditions, with higher $\mathrm{HF}: \mathrm{HNO}_{3}$ ratios and lower texturing temperatures resulting in deeper pits which are characterised by larger $h / D$ values. Figure 8 shows a comparison between simulated and experimental reflection of two textured wafers which had different $h / D$ values over the wavelength range of $250 \mathrm{~nm}$ to $1300 \mathrm{~nm}$. The simulated reflection data predicts the main trends in the experimental reflection curves, however, some inaccuracy in the simulated data is to be expected due to the simplified assumptions of the model (e.g., assumptions that the surface is characterised by a single $h / D$ value). Furthermore, mismatches between regions selected for reflection measurement and SEM imaging may contribute to discrepancies between simulated and experimental reflection values. Values of $h / D$ greater than 0.5 were not considered in this analysis because these surfaces involve more coupling of surrounding pits and possible porous silicon formation than surface morphologies that are typically observed with UKN texturing.

The relationship between the WAR and $h / D$ was also investigated, due to the usefulness of WAR values in comparing different texturing methods. Figure 9 shows how the WAR varies with $h / D$ values. The WAR does not vary significantly with increasing $h / D$ until an $h / D$ value of $\sim 0.2$, after which it begins to decrease and subsequently plateaus at a value of 0.24 . The decrease in WAR, which occurs at $\sim 0.2$ in Figure 9, is due to the onset of higher-order reflection (i.e., $\left.k_{m}=2\right)$. The critical value of $h / D$ can be calculated from (3) 


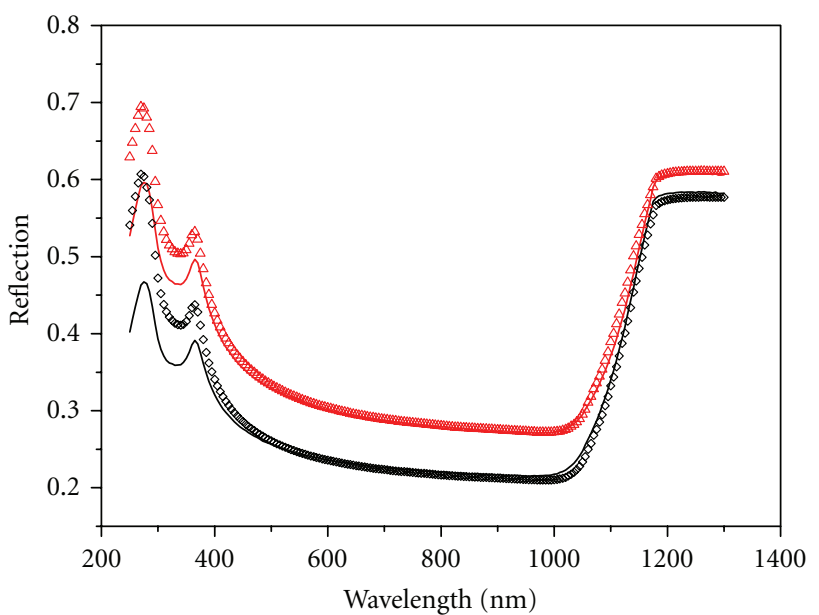

FIGURE 8: Simulated and measured reflection values graphed as a function of wavelength for two samples with different $h / D$ values. Reflection values for $h / D=0.25$ are shown in red with the solid line and (red triangle) symbols denoting the simulated and measured values, respectively. Reflection values for $h / D=0.37$ are shown in black with the solid line and $(\diamond)$ symbols denoting the simulated and measured values, respectively.

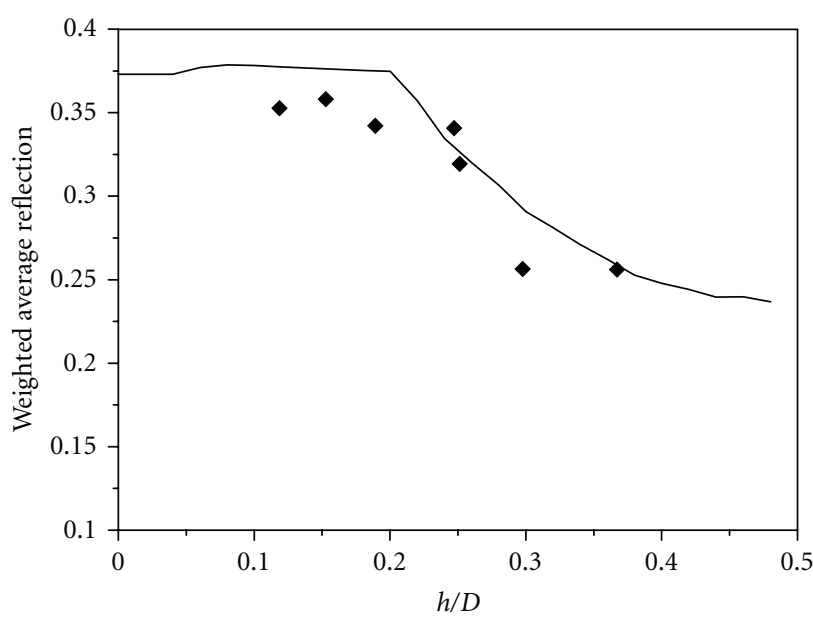

FIgURE 9: Simulated (solid line) and measured ( $\downarrow$ ) WAR values, computed using the wavelength range of $300 \mathrm{~nm}$ to $1200 \mathrm{~nm}$, as a function of $h / D$.

to be 0.2071 , after which the reflection is reduced due to the reduced front surface reflection.

The contribution of the rear surface reflection to the increased reflection predicted by the model and depicted in Figure 5 suggests that, at least for longer wavelengths when $h / D$ is small and light has a greater probability of reaching the rear surface because of the shorter light path in the wafer, some increase in reflection is expected. By graphing the simulated WAR of the short $(550-600 \mathrm{~nm})$ and long wavelengths (1150-1200 nm) of light separately, as shown in Figure 10, this predicted increase in reflection is demonstrated for only the longer wavelength light.

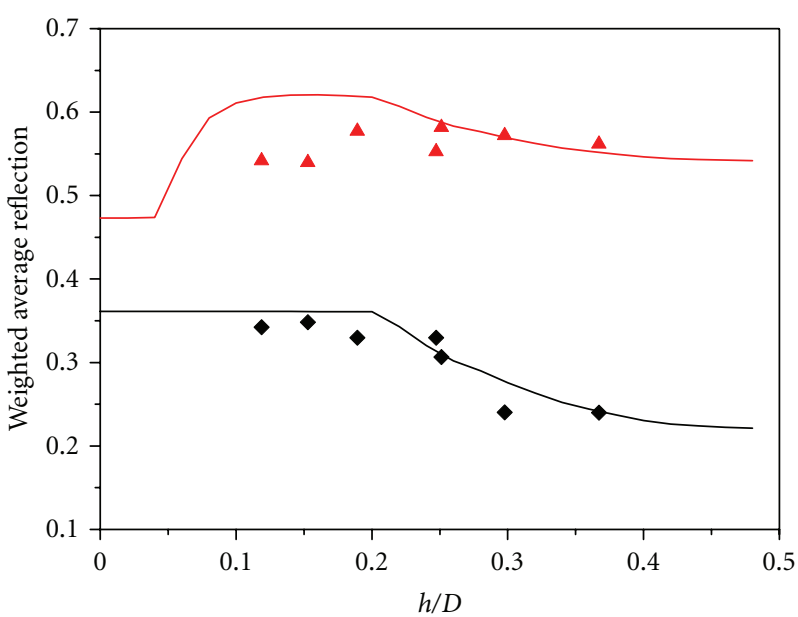

Figure 10: Simulated and measured WAR values for shortwavelength region ( $550 \mathrm{~nm}$ to $600 \mathrm{~nm}$ ) and long-wavelength region $(1150 \mathrm{~nm}$ to $1200 \mathrm{~nm})$ graphed as a function of $h / D$. The black solid line and $(\checkmark)$ values represent the simulated and measured values for the short wavelength region and the red solid line and (red triangle) symbols represent the simulated and measured values for the longwavelength region.

However, although the model predicts the WAR reasonably well for the short wavelength light where the reflection is largely determined by the front surface, it appears to only successfully predict the reflection of long wavelength light for $h / D$ values greater than $\sim 0.25$. For the cases where $h / D$ is higher than the critical value of high-order reflection, the WAR of both short- and long-wavelength light decreases as $h / D$ increases because the front surface reflection is effectively reduced by higher-order reflections within etching pits. However, as $h / D$ becomes even larger, higher-order reflection only occurs on a smaller surface of the etching pit, thus the value of the WAR plateaus as the value of $h / D$ approaches 0.5 . For the region where $h / D$ is lower than the critical value, the WAR of short wavelength light is substantially unchanged because there is no significant higher-order reflection arising from the front surface. The short wavelength light has a high probability of absorption before reaching the rear surface without requiring a lengthened light path due to front surface scattering.

Increased reflection for long wavelength light at low $h / D$ values is not evident in the experimental data. This is most likely due to higher-order reflection not being considered in the rear surface reflection model described in Section 2.3. This can result in underestimation of the light path length in the wafer and hence higher predicted WAR values for low $h / D$ values. However, the model appears to accurately predict the WAR for larger $h / D$ values, because the longer light paths caused by the increased scattering increases the probability that the light is absorbed in the bulk and therefore reduces the sensitivity of the model to the rear surface reflection as shown in Figure 5. For small $h / D$ values, light is coupled into the wafer at less oblique angles and hence experiences shorter light paths in the wafer and is less likely to be internally reflected. 


\section{Conclusions}

An optical model was presented for the modelling of light trapping in acidic-textured silicon wafers. Sphere caps were used to model the etching pits that form during the texturing when the commonly-used UKN texturing process is used. The developed model was used to predict the reflection from bare acidic-textured multicrystalline wafers on the basis of the surface morphology which was varied by altering the texturing conditions. The model was shown to predict the reflection for a wide range of wavelengths. The model predicted that, for shorter wavelength light, the WAR was constant for small $h / D$ values and then began to decrease at $h / D \sim 0.2$ where higher-order reflection effects became significant resulting in increased scattering of light in the wafer. For longer wavelength light, where the absorption coefficient is significantly reduced, the model predicted a small increase in WAR for small $h / D$ values due to the shorter paths experienced by light that is coupled less obliquely into the wafer. This results in a greater chance of initially captured light escaping from the wafer on subsequent encounters with wafer surfaces. However, this increase in WAR for low $h / D$ values was not observed experimentally. It is proposed that this was due to the fact that the rear surface reflection model used did not take into account the effects of higherorder reflection at the convex surface of the etching pits and thus, most likely, underestimated the length of the internally reflected light path.

The inaccuracies of the rear surface reflection model may not be critical for practical purposes because the rear surfaces of many acidic-textured silicon solar cells are partially planarised by a rear surface etching process used to isolate the junction to the front surface of the cell and then alloyed with aluminium to form a rear-surface field and rear-cell electrode. Consequently, the rear surface reflection model, developed here for bare wafers, will need to be further developed to more accurately represent the light trapping properties of final devices.

Finally, the model has the potential to be adapted for use in solar cell simulators, such as PC1D, or higherlevel simulators such as the VPL where it can be used to relate acidic texturing processing conditions to final device efficiency and hence enhance the educational and training experiences for future PV engineers. The accuracy of the model could be enhanced by an improved characterisation of the surface morphology of the textured surfaces which are formed using different processing parameters, and further characterisation of the reflectance from final devices.

\section{Acknowledgment}

The authors would like to thank Dr. Deming Zhu of UNSW Electron Microscopy Unit for assistance with scanning electron microscopy.

\section{References}

[1] E. Yablonovitch and G. D. Cody, "Intensity enhancement in textured optical sheets for solar cells," IEEE Transactions on Electron Devices, vol. 29, no. 2, pp. 300-305, 1982.
[2] P. Campbell and M. A. Green, "Light trapping properties of pyramidally textured surfaces," Journal of Applied Physics, vol. 62, no. 1, pp. 243-249, 1987.

[3] A. Wang, J. Zhao, and M. Green, "24-percent efficient silicon solar cells," Applied Physics Letters, vol. 57, pp. 602-604, 1990.

[4] J. Zhao, A. Wang, and P. Campbell, "A 19.8\% efficient honeycomb multicrystalline silicon solar cell with improved light trapping," IEEE Transactions on Electron Devices, vol. 46, no. 10, pp. 1978-1983, 1999.

[5] G. Willeke, H. Nussbaumer, H. Bender, and E. Bucher, "A simple and effective light trapping technique for polycrystalline silicon solar cells," Solar Energy Materials and Solar Cells, vol. 26, no. 4, pp. 345-356, 1992.

[6] C. Gerhards, C. Marckmann, R. Tolle et al., "Mechanically V-textured low cost multicrystalline silicon solar cells with a novel printing metallization," in Proceedings of the of the 26th IEEE Photovoltaic Specialists Conference, pp. 43-46, 1997.

[7] Y. Inomata, K. Fukui, and K. Shirasawa, "Surface texturing of large area multicrystalline silicon solar cells using reactive ion etching method," Solar Energy Materials and Solar Cells, vol. 48, no. 1-4, pp. 237-242, 1997.

[8] W. A. Nositschka, C. Beneking, O. Voigt, and H. Kurz, "Texturisation of multicrystalline silicon wafers for solar cells by reactive ion etching through colloidal masks," Solar Energy Materials and Solar Cells, vol. 76, no. 2, pp. 155-166, 2003.

[9] D. S. Ruby, S. H. Zaidi, S. Narayanan, B. M. Damiani, and A. Rohatgi, "Rie-texturing of multicrystalline silicon solar cells," Solar Energy Materials and Solar Cells, vol. 74, no. 1-4, pp. 133-137, 2002.

[10] J. C. Zolper, S. Narayanan, S. R. Wenham, and M. A. Green, " $16.7 \%$ efficient, laser textured, buried contact polycrystalline silicon solar cell," Applied Physics Letters, vol. 55, no. 22, pp. 2363-2365, 1989.

[11] M. Abbott and J. Cotter, "Optical and electrical properties of laser texturing for high-efficiency solar cells," Progress in Photovoltaics, vol. 14, no. 3, pp. 225-235, 2006.

[12] L. A. Dobrzański, A. Drygała, K. Gołombek, P. Panek, E. Bielańska, and P. Zieba, "Laser surface treatment of multicrystalline silicon for enhancing optical properties," Journal of Materials Processing Technology, vol. 201, no. 1-3, pp. 291-296, 2008.

[13] H. Seidel, L. Csepregi, A. Heuberger, and H. Baumgaertel, "Anisotropic etching of crystalline silicon in alkaline solutions. I. Orientation dependence and behavior of passivation layers," Journal of the Electrochemical Society, vol. 137, no. 11, pp. 3612-3626, 1990.

[14] Z. Xi, D. Yang, W. Dan, C. Jun, X. Li, and D. Que, "Investigation of texturization for monocrystalline silicon solar cells with different kinds of alkaline," Renewable Energy, vol. 29, no. 13, pp. 2101-2107, 2004.

[15] J. D. Hylton, A. R. Burgers, and W. C. Sinke, "Alkaline etching for reflectance reduction in multicrystalline silicon solar cells," Journal of the Electrochemical Society, vol. 151, no. 6, pp. G408G427, 2004.

[16] R. Einhaus, E. Vazsonyi, J. Szlufcik, J. Nijs, and R. Mertens, "Isotropic texturing of multicrystalline silicon wafers with acidic texturing solutions," in Proceedings of the IEEE 26th Photovoltaic Specialists Conference, pp. 167-170, October 1997.

[17] K. Kim, S. K. Dhungel, S. Jung, D. Mangalaraj, and J. Yi, “Texturing of large area multi-crystalline silicon wafers through different chemical approaches for solar cell fabrication," Solar Energy Materials and Solar Cells, vol. 92, no. 8, pp. 960-968, 2008. 
[18] A. Hauser, I. Melnyk, E. Wefringhaus, F. Delahaye, G. Vilsmeier, and P. Fath, "Acidic texturisation of mc-Si using a high throughput in-line prototype system with no th organic chemistry," in Proceedings of the 19th European Photovoltaic Solar Energy Conference, pp. 1094-1097, 2004.

[19] S. Mathijssen, S. Braun, I. Melnyk et al., "Survey of acid texturing and new innovative acid processes for mc solar wafers," in Proceedings of the 24th European Photovoltaic Solar Energy Conference, pp. 1964-1967, 2009.

[20] A. Hauser, I. Melnyk, P. Fath, S. Narayanan, S. Roberts, and T. M. Bruton, "A simplified process for isotropic texturing of MC-SI," in Proceddings of the 3rd World Conference on Photovoltaic Energy Conversion, vol. 2, pp. 1447-1450, May 2003.

[21] Z. Xi, D. Yang, W. Dan, C. Jun, X. Li, and D. Que, "Texturization of cast multicrystalline silicon for solar cells," Semiconductor Science and Technology, vol. 19, no. 3, pp. 485-489, 2004.

[22] Y. Nishimoto, T. Ishihara, and K. Namba, "Investigation of acidic texturization for multicrystalline silicon solar cells," Journal of the Electrochemical Society, vol. 146, no. 2, pp. 457461, 1999.

[23] J. I. Owen, J. Hüpkes, H. Zhu, E. Bunte, and S. E. Pust, "Novel etch process to tune crater size on magnetron sputtered ZnO:Al," Physica Status Solidi A, vol. 208, no. 1, pp. 109-113, 2011.

[24] H. Zhang, L. Zheng, X. Ma, B. Zhao, C. Wang, and F. Xu, "Nucleation and bulk growth control for high efficiency silicon ingot casting," Journal of Crystal Growth, vol. ¿318, no. 1, pp. 283-287, 2011.

[25] K. Fujiwara, W. Pan, N. Usami et al., "Growth of structurecontrolled polycrystalline silicon ingots for solar cells by casting," Acta Materialia, vol. 54, no. 12, pp. 3191-3197, 2006.

[26] N. Stoddard, B. Wu, I. Witting et al., "Casting single crystal silicon: novel defect profiles from BP solar's mono2 wafers," Solid State Phenomena, vol. 131-133, pp. 1-8, 2008.

[27] D. A. Clugston and P. A. Basore, "PC1D version 5: 32-bit solar cell modeling on personal computers," in Proceedings of the IEEE 26th Photovoltaic Specialists Conference, pp. 207-210, October 1997.

[28] S. R. Wenham and A. Bruce, "Virtual production line for the manufacturing of screen-printed solar cells," in Proceedings of the 19th European Photovoltaic Solar Energy Conference, 2004.

[29] Z. Li, Y. Li, M. J. Fu, and A. Lennon, "Simulation of acidic texturing for the virtual production line software," in Proceedings of the 21st International Photovoltaic Science and Engineering Conference, 2011.

[30] Y. T. Cheng, J. J. Ho, W. J. Lee et al., "Investigation of low-cost surface processing techniques for large-size multicrystalline silicon solar cells," International Journal of Photoenergy, vol. 2010, Article ID 268035, 6 pages, 2010.

[31] M. A. Green and M. J. Keevers, "Optical properties of intrinsic silicon at $300 \mathrm{~K}$," Progress in Photovoltaics, vol. 3, no. 3, pp. 189-192, 1995. 


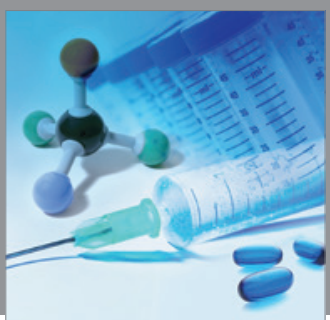

International Journal of

Medicinal Chemistry

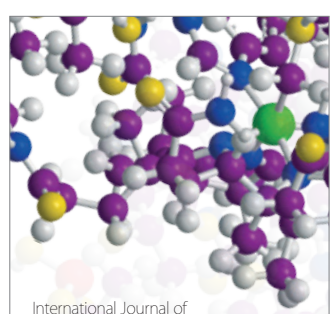

Carbohydrate Chemistry

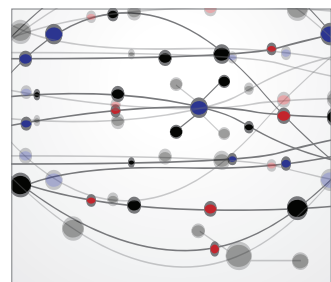

The Scientific World Journal
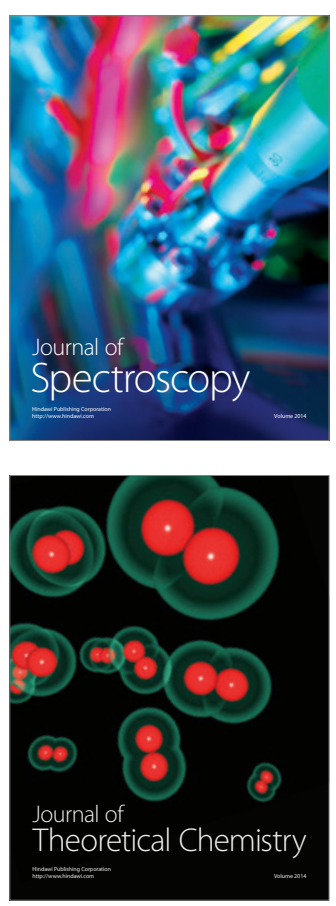
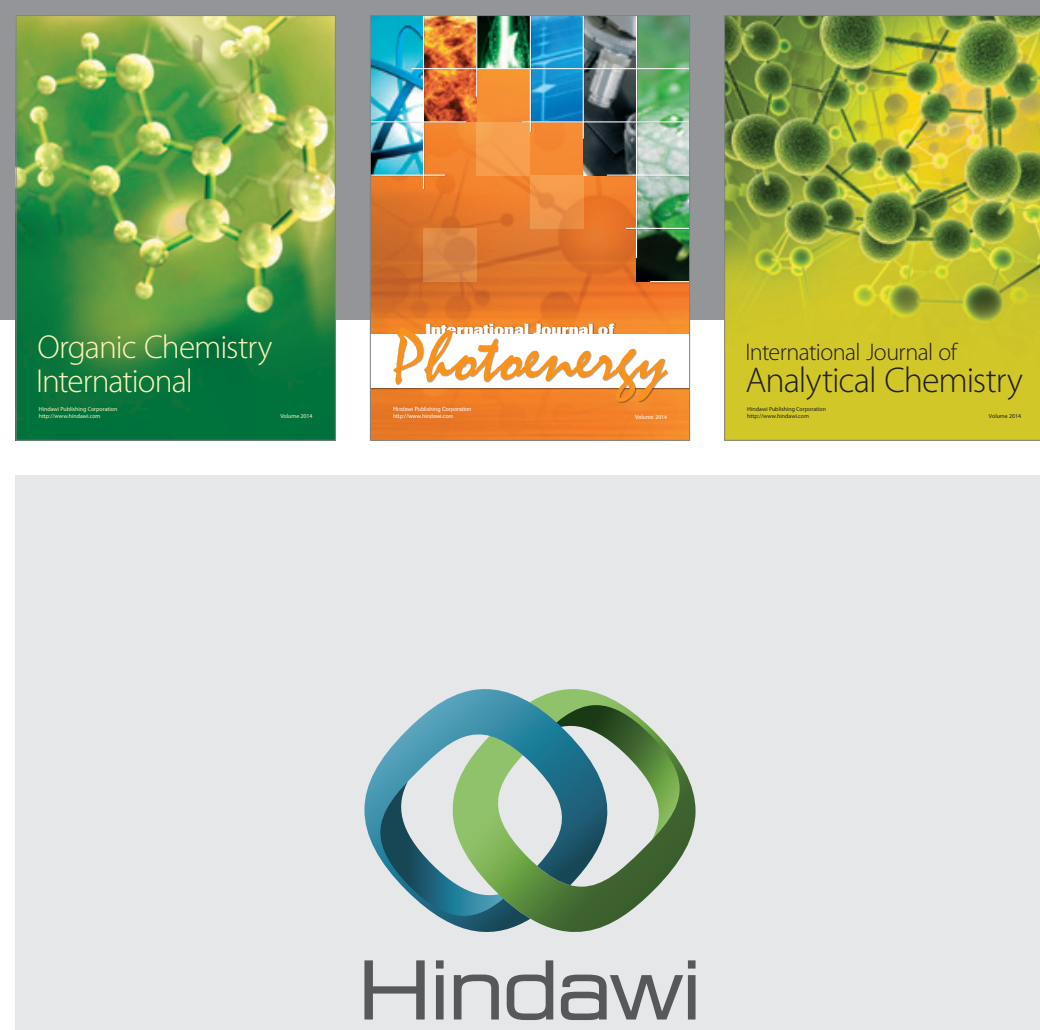

Submit your manuscripts at

http://www.hindawi.com
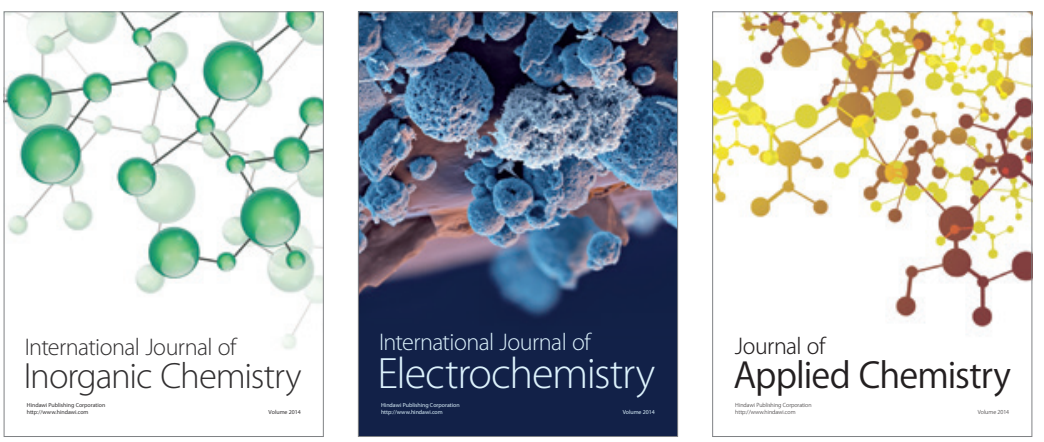

Journal of

Applied Chemistry
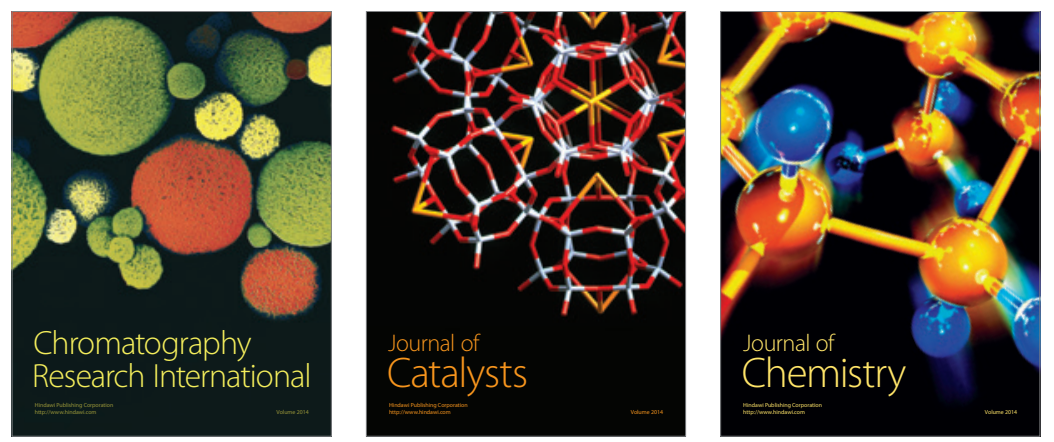
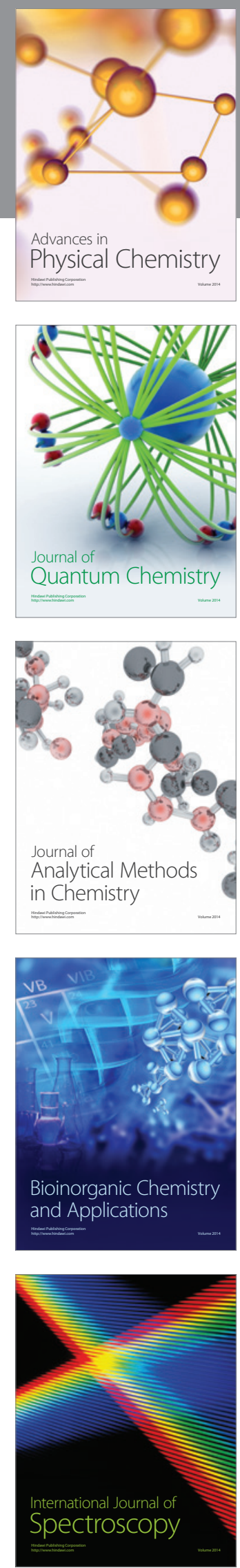